\title{
Advanced glycation end-products induce basement membrane hypertrophy in endoneurial microvessels and disrupt the blood-nerve barrier by stimulating the release of TGF- $\beta$ and vascular endothelial growth factor (VEGF) by pericytes
}

\author{
F. Shimizu $\cdot$ Y. Sano $・$ H. Haruki $\cdot$ T. Kanda
}

Received: 21 August 2010 / Accepted: 7 February 2011 / Published online: 16 March 2011

(C) Springer-Verlag 2011

\begin{abstract}
Aims/hypothesis The breakdown of the blood-nerve barrier (BNB) is considered to be a key step in diabetic neuropathy. Although basement membrane hypertrophy and breakdown of the BNB are characteristic features of diabetic neuropathy, the underlying pathogenesis remains unclear. The purpose of the present study was to identify the possible mechanisms responsible for inducing the hypertrophy of basement membrane and the disruption of the BNB after exposure to AGEs.

Methods The newly established human peripheral nerve microvascular endothelial cell (PnMEC) and pericyte cell lines were used to elucidate which cell types constituting the BNB regulate the basement membrane and to investigate the effect of AGEs on the basement membrane of the BNB using western blot analysis.

Results Fibronectin, collagen type IV and tissue inhibitor of metalloproteinase (TIMP-1) protein were produced mainly by peripheral nerve pericytes, indicating that the basement membrane of the BNB is regulated mainly by these cells. AGEs reduced the production of claudin-5 in PnMECs by increasing autocrine signalling through vascular endothelial growth factor (VEGF) secreted by the PnMECs themselves.
\end{abstract}

Electronic supplementary material The online version of this article (doi:10.1007/s00125-011-2107-7) contains supplementary material, which is available to authorised users.

F. Shimizu $\cdot$ Y. Sano $\cdot$ H. Haruki $\cdot$ T. Kanda $(\bowtie)$

Department of Neurology and Clinical Neuroscience,

Yamaguchi University Graduate School of Medicine,

1-1-1, Minami kogushi,

Ube, Yamaguchi 7558505, Japan

e-mail: tkanda@yamaguchi-u.ac.jp
Furthermore, AGEs increased the amount of fibronectin, collagen type IV and TIMP-1 in pericytes through a similar upregulation of autocrine VEGF and transforming growth factor (TGF) $-\beta$ released by pericytes.

Conclusions/interpretation These results indicate that pericytes may be the main regulators of the basement membrane at the BNB. AGEs induce basement membrane hypertrophy and disrupt the BNB by increasing autocrine VEGF and TGF- $\beta$ signalling by pericytes under diabetic conditions.

Keywords Advanced glycation end-products · Basement membrane hypertrophy $\cdot$ Blood-nerve barrier .

Diabetic neuropathy

\begin{tabular}{ll}
\multicolumn{2}{l}{ Abbreviations } \\
Act- & Active- \\
AGEs & Advanced glycation end-products \\
BBB & Blood-brain barrier \\
bFGF & Basic fibroblast growth factor \\
BNB & Blood-nerve barrier \\
BRB & Blood-retinal barrier \\
ECM & Extracellular matrix \\
GAPDH & Glyceraldehyde-3-phosphate dehydrogenase \\
MMP & Matrix metalloproteinase \\
NF-kB & Nuclear factor $k$ B \\
PnMECs & Peripheral nerve microvascular endothelial cells \\
RAGE & Receptor of advanced glycation end-product \\
TEER & Transendothelial electrical resistance \\
TGF & Transforming growth factor \\
TIMP & Tissue inhibitor of metalloproteinase \\
VEGF & Vascular endothelial growth factor
\end{tabular}




\section{Introduction}

Diabetic neuropathy is one of the most frequent complications of diabetes. Microangiopathy of the endoneurium is a common finding in the nerves of diabetic patients, and is thought to be a contributing factor to the development of diabetic neuropathy. The blood-nerve barrier (BNB) is localised in the microvessels of the endoneurium or perineurium, and consists of peripheral nerve microvascular endothelial cells (PnMECs), pericytes of endoneurial microvascular origin, and the basement membrane [1-3]. The basement membrane of the BNB plays important roles in maintaining the structure and function of capillary vessels [4]. Recently, breakdown of the BNB has been suggested to be an initial key step in diabetic neuropathy [4-6]. Although hypertrophy of the basement membrane in the endoneurium or perineurium, pericyte loss and endothelial cell hyperplasia at the $\mathrm{BNB}$ are characteristic features of diabetic neuropathy [6], the pathology and pathogenesis remain unclear. It is known, however, that the basement membrane is constructed from extracellular matrix (ECM) components such as collagen type IV, laminin and fibronectin, and is regulated by matrix metalloproteinases (MMPs) at the blood-brain barrier (BBB) [7, 8]. MMP-9 and MMP-2 digest collagen type IV, fibronectin and laminin, and are inhibited by tissue inhibitor of metalloproteinase (TIMP) [9]. Several studies have suggested that brain pericytes or astrocytes synthesise fibronectin, MMP-2 and MMP-9, and regulate the state of the basement membrane in the $\mathrm{BBB}[10$, 11]. However, the mechanisms underlying in the formation and maintenance of the basement membrane in the BNB have not yet been elucidated.

Hyperglycaemia is responsible for the presence of high levels of non-enzymatically produced AGEs in the diabetic patients [12]. Their accumulation on proteins in the microvasculature appears to be a key factor in the development of retinopathy and nephropathy [12], but their role remains unclear with regard to their importance in diabetic neuropathy. AGEs have been reported to directly stimulate the production of basement membrane by activating transforming growth factor (TGF)- $\beta 1$ secretion during the development of diabetic nephropathy [13], and to induce blood-retinal barrier (BRB) dysfunction through vascular endothelial growth factor (VEGF) production in diabetic retinopathy [14]. However, to date, the molecular mechanism by which AGEs induce hypertrophy of the basement membrane and breakdown of the BNB during diabetic neuropathy is unclear. The purpose of this study was to elucidate which type of cells regulate the basement membrane of the BNB using our newly developed PnMEC and pericyte cell lines, and to identify the possible mechanisms responsible for increasing the basement membrane thickness of the BNB after exposure to AGEs.

\section{Methods}

Reagents The culture medium for pericytes and PnMECs consisted of DMEM (Sigma, St Louis, MO, USA) containing $100 \mathrm{U} / \mathrm{ml}$ penicillin (Sigma), $100 \mu \mathrm{g} / \mathrm{ml}$ streptomycin (Sigma), $25 \mathrm{ng} / \mathrm{ml}$ amphotericin B (Invitrogen, Grand Island, NY, USA) and 10\% fetal bovine serum (FBS) (Sigma). Polyclonal anti-TIMP-1, anti-fibronectin, antiMMP-2, and anti-MMP-9 antibodies were obtained from Santa Cruz Biotechnology (Santa Cruz, CA, USA). Polyclonal anti-claudin-5 and anti-occludin antibodies were purchased from Zymed (San Francisco, CA, USA). Polyclonal anti-collagen type IV was obtained from ARP American Research Products (Belmont, MA, USA). AGEs-BSA was purchased from Calbiochem (Darmstadt, Germany). Polyclonal anti-basic fibroblast growth factor (bFGF), anti-TGF- $\beta$ and anti-VEGF antibodies were purchased from R\&D Systems (Minneapolis, MN, USA). Human astrocytes were purchased from Lonza (Walkersville, MD, USA).

Cell culture The immortalised human PnMECs, and brain and peripheral nerve pericyte cell lines were generated as described previously by our group [15, 16]. The study protocol for human tissue was approved by the ethics committee of the Medical Faculty, University of Yamaguchi Graduate School, and was conducted in accordance with the Declaration of Helsinki, as amended in Somerset West in 1996. Written informed consent was obtained from the families of the participants before entering the study. The cell lines, which were isolated from human sciatic nerve and brain tissue, retain the morphological characteristics of primary peripheral nerve endothelial cells or brain and peripheral nerve pericytes and produce either endothelial or pericyte markers. The cultures were maintained at $37^{\circ} \mathrm{C}$ in $5 \%$ (vol./vol.) $\mathrm{CO}_{2}$ and the DMEM medium was replaced every 3 days.

Quantitative real-time PCR analysis Total RNA was extracted using an RNeasy Plus Mini Kit (Qiagen, Hilden, Germany). Single-stranded cDNA was created from $40 \mathrm{ng}$ total RNA using the StrataScript First Strand Synthesis System (Stratagene, Cedar Creek, TX, USA). The sequence specificity of each human primer pair is shown in the electronic supplementary material (ESM) Table 1. Quantitative real-time PCR analysis was performed using the Mx3005P (Stratagene) with FullVelocity SYBR Green QPCR master mix (Stratagene). Glyceraldehyde-3phosphate dehydrogenase $(G A P D H)$ was used as an internal standard. The samples were subjected to PCR analysis using the following cycling parameters: $95^{\circ} \mathrm{C}$ for $10 \mathrm{~min}, 95^{\circ} \mathrm{C}$ for $15 \mathrm{~s}$ and $60^{\circ} \mathrm{C}$ for $1 \mathrm{~min}$ for 40 cycles. Negative controls (cDNA-free solutions) were included in 
Table 1 Effect of the two AGE concentrations (100 and $200 \mu \mathrm{g} / \mathrm{ml}$ ) on production of basement-membrane-related molecules in peripheral nerve pericytes

\begin{tabular}{lll}
\hline Protein & \multicolumn{2}{l}{ Ratio of target protein production (protein:actin) } \\
\cline { 2 - 3 } & AGE $100 \mu \mathrm{g} / \mathrm{ml}$ & AGE $200 \mu \mathrm{g} / \mathrm{ml}$ \\
\hline Collagen type IV & $2.18 \pm 0.39^{* *}$ & $2.93 \pm 0.61^{* *}$ \\
Fibronectin & $4.83 \pm 0.52^{* *}$ & $5.33 \pm 0.96^{* *}$ \\
TIMP-1 & $2.38 \pm 0.17^{* *}$ & $2.86 \pm 0.14^{* *}$ \\
Act-MMP-9 & $0.68 \pm 0.06$ & $0.36 \pm 0.18^{* *}$ \\
Act-MMP-2 & $1.15 \pm 0.07$ & $0.94 \pm 0.05$ \\
RAGE & $2.03 \pm 0.38^{* *}$ & $2.40 \pm 0.42^{* *}$ \\
TGF- $\beta$ & $1.87 \pm 0.13^{* *}$ & $2.68 \pm 0.18^{* *}$ \\
VEGF & $2.26 \pm 0.29 * *$ & $2.21 \pm 0.21^{* *}$ \\
bFGF & $1.18 \pm 0.08$ & $0.96 \pm 0.05$ \\
\hline
\end{tabular}

Data shown are means $\pm \operatorname{SEM}(n=3)$

Each value reflects the combined densitometry data from three independent experiments and is shown as a fold increase above control

$* * p<0.01$ compared with control

each reaction. The standard reaction curve $\left(R_{\mathrm{v}}\right)$ was analysed using MxPro software (Stratagene), with the relative quantity was calculated according to the formula $R_{\mathrm{v}}=R_{\text {gene }} R_{\text {gene }} / R_{\mathrm{GAPDH}}$ by the software.

Western blot analysis The protein samples (10-20 $\mu \mathrm{g})$ were separated by SDS-PAGE (Bio-Rad), and transferred to nitrocellulose membrane (Amersham, Chalfont, UK). The membranes were treated with blocking buffer ( $5 \%$ skimmed milk in $25 \mathrm{mmol} / \mathrm{l}$ Tris- $\mathrm{HCl} \mathrm{pH} 7.6,125 \mathrm{nmol} / \mathrm{l} \mathrm{NaCl}, 0.5 \%$ Tween 20) for $1 \mathrm{~h}$ at room temperature and incubated with relevant primary antibodies (dilution 1:100) for $2 \mathrm{~h}$ at room temperature as the primary antibodies. The membrane was exposed to peroxidase-conjugated secondary antibody $(1: 2,000)$ followed by chemiluminescence reagent (Amersham), exposure to X-Omat $\mathrm{S}$ films (Amersham) and quantification of bands intensity using the Fuji image analysis software package.

Analysis of the effect of TGF- $\beta 1$ or VEGF on the expression of basement-membrane-related genes Peripheral nerve pericytes were either left untreated or treated with human TGF- $\beta 1(10 \mathrm{ng} / \mathrm{ml})$ or VEGF $(10 \mathrm{ng} / \mathrm{ml})$. The total protein was collected 2 days later.

Treatment of human PnMECs and peripheral nerve pericytes with $A G E s-B S A$ PnMECs and peripheral nerve pericytes were cultured in DMEM with unmodified BSA $(100 \mu \mathrm{g} / \mathrm{ml})$ and AGE-modified BSA $(100 \mu \mathrm{g} / \mathrm{ml}$, $200 \mu \mathrm{g} / \mathrm{ml}$ ). AGEs-BSA contained $<0.2 \mathrm{ng} / \mathrm{ml}$ of endotoxin. The cells were cultured for 2 days prior to collection of total protein.

VEGF or TGF- $\beta$ inhibitory study Peripheral nerve pericytes were cultured with AGEs-BSA containing $2.0 \mu \mathrm{g} / \mathrm{ml}$ antibody against VEGF or TGF- $\beta$, or normal rabbit IgG. PnMECs were also cultured with AGEs containing $2.0 \mu \mathrm{g} / \mathrm{ml}$ antibody against VEGF or normal rabbit IgG. The total RNA was extracted $24 \mathrm{~h}$ later, and total protein was obtained a further 2 days later.

Transendothelial electrical resistance (TEER) study Transwell inserts (pore size $0.4 \mu \mathrm{m}$, effective growth area $0.3 \mathrm{~cm}^{2}$; BD Biosciences, Franklin Lakes, NJ, USA) were coated by rat-tail collagen type-I (BD Biosciences). TEER values of cell layers were measured with a Millicell electrical resistance apparatus (Endohm-6 and EVOM, World Precision Instruments, Sarasota, FL, USA). The PnMECs were seeded $\left(1 \times 10^{6}\right.$ cells per insert $)$ on the upper compartment and cultured for $24 \mathrm{~h}$. In order to estimate the effect of AGEs against PnMECs, the upper compartment was incubated with each medium (control, AGE, AGE+ VEGF antibody) for $24 \mathrm{~h}$.

Data analysis Unless otherwise indicated, all data represent the mean \pm SEM. An unpaired two-tailed Student's $t$ test was used to determine the significance of differences between two group means. A $p$ value $<0.01$ was considered to be statistically significant.

\section{Results}

The expression of basement-membrane-related molecules secreted from the peripheral nerve pericytes We quantified the mRNA expression of fibronectin, collagen type IV (COL4A4), TIMP1, MMP2 and MMP9 by means of relative quantification with real-time RT-PCR using PnMECs and peripheral nerve pericytes (ESM Fig. 1a-e). The expression of COL4A4 (ESM Fig. 1a), fibronectin (ESM Fig. 1b) and TIMP1 (ESM Fig. 1c) mRNA in peripheral nerve pericytes was significantly higher than those in PnMECs $(p<0.01)$. There were no significant differences in expression of MMP2 (ESM Fig. 1d) and MMP9 (ESM Fig. 1e) between these two cells. In addition, we determined fibronectin, collagen type IV, TIMP-1, MMP-2 and MMP-9 protein production using PnMECs and peripheral nerve cells by a western blot analysis (Fig. 1a). The fibronectin, collagen type IV, TIMP-1, MMP-2, and MMP-9 bands, corresponding to 190, 25, 38, and $92 \mathrm{kDa}$ single bands, respectively, were detected in these cell lines (Fig. 1a). Although the collagen type IV and 


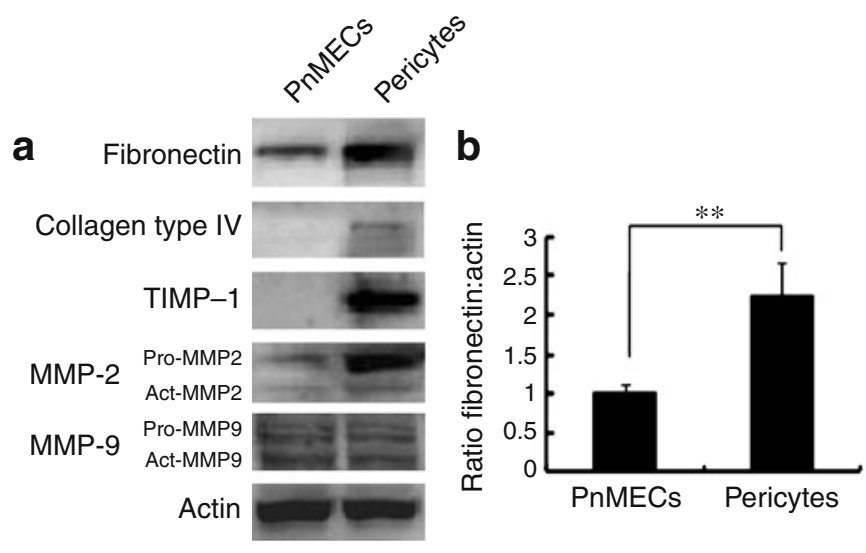

Fig. 1 a Western blot analysis of collagen type IV, fibronectin, TIMP-1, MMP-2 and MMP-9 proteins in PnMECs and peripheral nerve pericytes. Fibronectin, collagen type IV, TIMP-1, MMP-2 and MMP-9 bands corresponded to single bands at $190,25,38$, and $92 \mathrm{kDa}$, respectively. Bands corresponding to collagen type IV and TIMP-1 were not detected in PnMECs, although the fibronectin, MMP-2 and MMP-9 proteins were

TIMP-1 bands were not detected, the fibronectin, MMP-2, and MMP-9 proteins were produced in PnMECs (Fig. 1a). Interestingly, the production of fibronectin (Fig. 1b) and MMP-2 (Fig. 1c) proteins in the peripheral nerve pericytes was significantly higher than those in PnMECs $(p<0.01)$, whereas production of MMP-9 protein production did not show a significant difference between these two cells (Fig. 1d).

Effect of AGEs on the production of basement-membranerelated molecules in peripheral nerve pericytes To identify the possible mechanism responsible for the basement membrane hypertrophy of the BNB after exposure to AGEs, we also examined the changes in the production of fibronectin, collagen type IV, TIMP-1, MMP-2 and MMP-9 proteins in peripheral nerve pericytes after AGE exposure $(100,200 \mu \mathrm{g} / \mathrm{ml}$; Fig. 2a). The production of fibronectin and collagen type IV protein in peripheral nerve pericytes significantly increased in a dose-dependent manner after exposure to AGEs $(p<0.01$; Table 1$)$. The TIMP-1 protein production in peripheral nerve pericytes was also significantly increased after application of AGEs; however, the production of active MMP-9 (act-MMP-9) was decreased by treatment with $200 \mu \mathrm{g} / \mathrm{ml}$ AGE (Table 1). The level of the receptor of AGE (RAGE) in peripheral nerve pericytes was observed to increase significantly after incubation with AGEs in a dose-dependent manner (Table 1). We previously reported that our peripheral nerve pericytes released several growth factors, including VEGF, TGF- $\beta$ and bFGF, to maintain peripheral nerve homeostasis at the BNB [15]. We also quantified the change of these growth factors in
C

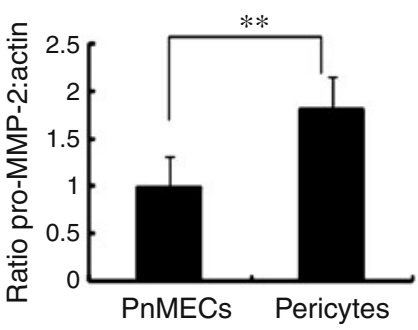

d

visualised. b-d The bar graph reflects the combined densitometry data from five independent experiments (mean \pm SEM, $n=5)$. The production of fibronectin (b) and pro-MMP-2 (c) in peripheral nerve pericytes was significantly higher than in PnMECs, whereas production of pro-MMP-9 protein (d) did not show a significant difference between these two cell lines. ${ }^{* *} p<0.01$

peripheral nerve pericytes after incubation with AGEs (100 and $200 \mu \mathrm{g} / \mathrm{ml}$ ) by a western blot analysis (Fig. 2b). The production of VEGF and TGF- $\beta$ protein in peripheral nerve pericytes was significantly increased by treatment with AGEs, although there was no change in the bFGF protein production after AGE exposure (Table 1).

Contribution of TGF- $\beta 1$ and VEGF to the induction of basement-membrane-related molecules after AGE exposure TGF- $\beta 1$ and VEGF are known to be major mediators of the early and late vascular changes in diabetic microangiopathy. To investigate the effect of TGF- $\beta 1$ and VEGF on the production of basementmembrane-related molecules at the BNB, we also quantified the changes in the production of fibronectin, collagen type IV, TIMP-1, MMP-2 and MMP-9 in peripheral nerve pericytes after incubation with TGF$\beta 1(10 \mathrm{ng} / \mathrm{ml})$ and VEGF $(10 \mathrm{ng} / \mathrm{ml})$ by western blot analysis (Fig. 3). Fibronectin and collagen type IV in peripheral nerve pericytes significantly increased after exposure to TGF- $\beta 1$ or VEGF $(p<0.01$; Table 2$)$. The promatrix metalloproteinase (pro-MMP-9) protein in peripheral nerve pericytes was also upregulated $(p<0.01)$ after application of TGF- $\beta 1$ or VEGF (Table 2$)$. To clarify the individual contributions of VEGF and TGF$\beta 1$ to the increase in basement-membrane thickness after exposure to AGEs, the activities of VEGF and TGF- $\beta$ were neutralised using an anti-VEGF and anti-TGF- $\beta$ antibody. The production of fibronectin and collagen type IV protein in peripheral nerve pericytes was decreased after incubation with AGEs pretreated with the anti-TGF- $\beta$ 
Fig. 2 a The changes in collagen type IV, fibronectin, TIMP-1, MMP-2 and MMP-9 production in peripheral nerve pericytes after treatment with the two AGE concentrations (100 and $200 \mu \mathrm{g} / \mathrm{ml})$. The production of fibronectin, collagen type IV, TIMP-1 and RAGE in peripheral nerve pericytes was significantly increased after treatment with AGEs in a dose-dependent manner. The level of act-MMP-9 proteins in peripheral nerve pericytes was significantly reduced, whereas act-MMP-2 protein did not show a significant difference following AGE treatment. b The changes in TGF- $\beta$, VEGF and bFGF production in peripheral nerve pericytes treated with AGEs. The production of TGF- $\beta$ and VEGF in peripheral nerve pericytes was significantly increased after treatment with AGEs, whereas bFGF production was not affected following AGE treatment. Control, conditioned medium of unmodified BSA; AGE, condition medium of AGEs-BSA a

Collagen type IV

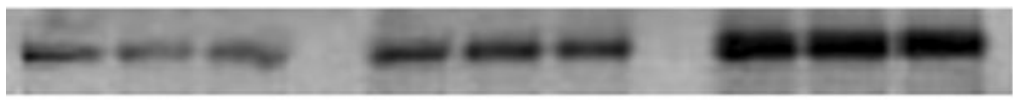

Fibronectin

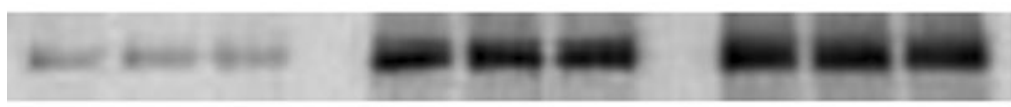

TIMP-1

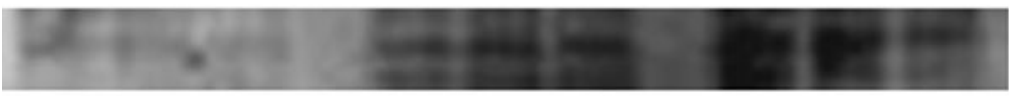

MMP-2

Pro-MMP2

Ac-MMP2

MMP-9

Pro-MMP9

Act-MMPg

RAGE

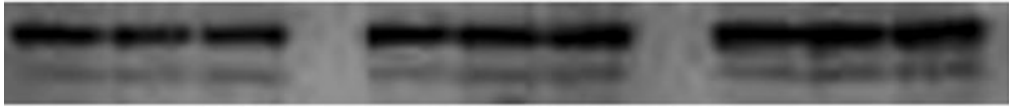

Actin
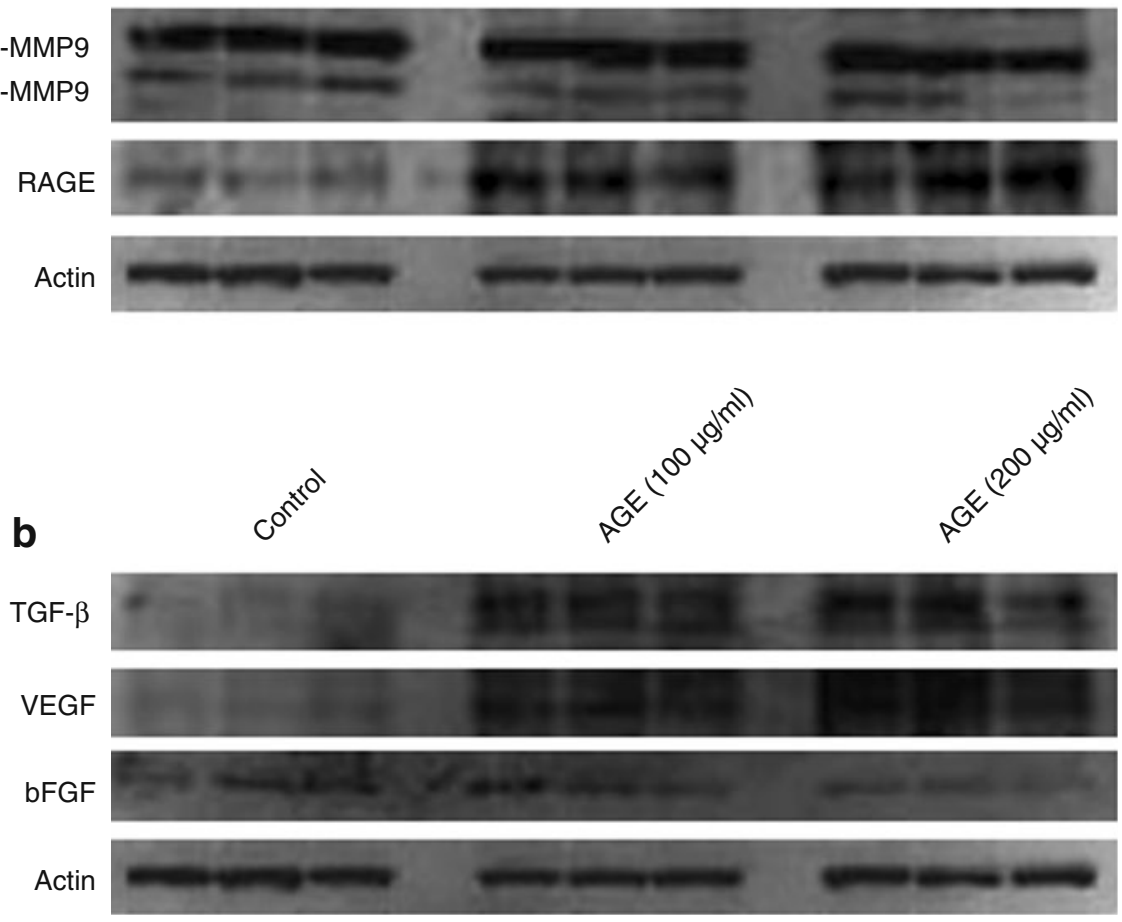

(Fig. 4a-c) or VEGF (Fig. 4d-f) antibody, as determined by a western blot analysis.

Effect of AGEs on the production of tight junctional molecules by PnMECs, and the contribution of VEGF to the induction of claudin-5 after AGE exposure To determine the effect of AGEs on the barrier function of the BNB, we also examined the changes in claudin-5 production by PnMECs after exposure to AGE $(100 \mu \mathrm{g} / \mathrm{ml})$ by a western blot analysis (Fig. 5a). Whereas the claudin-5 production in PnMECs was significantly reduced (Fig. 5b), VEGF production was significantly increased after incubation with AGEs (Fig. 5c). The TEER value of PnMECs did not change after incubation with AGEs (Fig. 5h). To clarify the contribution of VEGF to the reduction of claudin-5 in PnMECs, a neutralising anti-VEGF antibody was used. Relative quantification with real-time RT-PCR revealed that the percentage of increase in the claudin-5 (CLDN5) mRNA expression was $48 \%$ after incubation with AGEs pretreated using the anti-VEGF antibody (Fig. 5e). In addition, we quantified claudin-5 protein in PnMECs after incubation with the anti-VEGF neutralising antibody (Fig. 5f). Production of claudin-5 protein was increased after pre-incubation with the anti-VEGF neutralising antibody (Fig. $5 \mathrm{~g}$ ). The TEER value of PnMECs was also significantly increased after exposure to AGEs in cells pretreated with the anti-VEGF neutralising antibody (Fig. 5h). RAGE production in PnMECs 


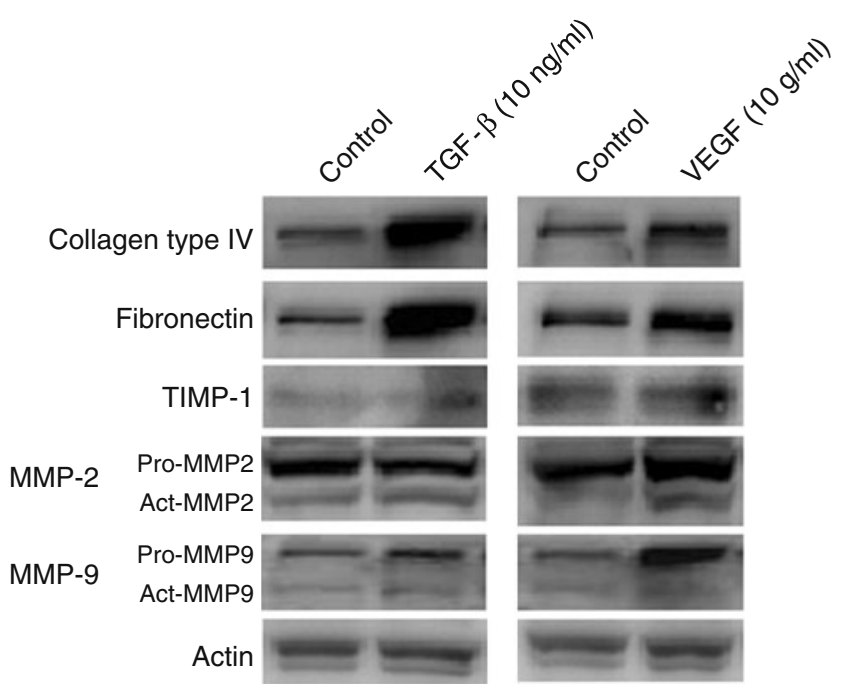

Fig. 3 The effect of VEGF $(10 \mathrm{ng} / \mathrm{ml})$ and TGF- $\beta(10 \mathrm{ng} / \mathrm{ml})$ on production of collagen type IV, fibronectin, TIMP-1, MMP-2, and MMP-9 proteins in peripheral nerve pericytes. The production of collagen type IV and fibronectin in peripheral nerve pericytes was significantly increased after treatment with TGF- $\beta$. The pro-MMP-9 proteins in peripheral nerve pericytes were upregulated after incubation with TGF- $\beta$, although TIMP-1 production was not affected. The production of collagen type IV and fibronectin in peripheral nerve pericytes was significantly increased after treatment with VEGF. The pro-MMP-9 proteins in peripheral nerve pericytes were upregulated after incubation with VEGF, although TIMP-1 production was not affected

was significantly induced after the application of AGEs (Fig. 5d).

\section{Discussion}

In this study, we examined the importance of the cells comprising the BNB in the formation or maintenance of the basement membrane. We have successfully established PnMEC and peripheral nerve pericyte cell lines of human origin $[15,16]$. Therefore, our in vitro BNB model is considered suitable for the analysis of the production of basement-membrane-related molecules, because it is difficult to identify cells that synthesise these molecules in vivo. Our study demonstrated that fibronectin, collagen type IV, MMP-2 and TIMP-1 are mainly synthesised by peripheral nerve pericytes, confirming that the maintenance of the basement membrane components is regulated primarily by peripheral nerve pericytes at the BNB. The basement membrane of the BBB is constructed from ECM-related molecules, including collagen type IV and fibronectin, and is regulated by TIMP-1, which inhibits MMPs [8]. Several studies suggest that astrocytes and brain pericytes synthesise fibronectin and collagen type IV, and these cells secrete
MMP-2 and MMP-9 to maintain basement membrane turnover $[11,17]$. Our study verified that peripheral nerve pericytes, which are the only cells in the endoneurial microvessels other than PnMECs, might be the key cells with regard to the maintenance of the basement membrane at the BNB.

The breakdown of the BNB is considered to be a key step in diabetic neuropathy $[5,6]$. Investigations of biopsied nerves with mild to severe diabetic neuropathy showed structural changes in the microvasculature in the endoneurium, including an increase in the basement membrane thickness, pericyte degeneration and endothelial hyperplasia [6]. Although basement membrane hypertrophy at the $\mathrm{BNB}$ is one of the cardinal pathogenic features of diabetic neuropathy [6], its pathogenesis remains unclear. TGF- $\beta 1$ is generally accepted to be the main pro-fibrotic factor in diabetic nephropathy [13]. Several lines of experimental and clinical evidence support a major role for TGF- $\beta 1$ in the development of glomerulosclerosis and interstitial fibrosis in diabetic nephropathy $[18,19]$. In another study, inhibition of TGF- $\beta 1$ with neutralising antibodies prevented the glomerular enlargement and the excess matrix production by reducing collagen type IV and fibronectin [20, 21]. Conversely, VEGF is also known to be another mediator of the early and late vascular changes in diabetic nephropathy and retinopathy. Several studies have suggested that VEGF signalling may affect renal matrix accumulation, because inhibition of VEGF with neutralising antibodies attenuates the increase in glomerular basement membrane thickness and mesangial matrix expansion in diabetic nephropathy [13, 22]. Furthermore, recent evidence has shown that VEGF may contribute to the breakdown of the BRB during diabetic retinopathy because

Table 2 Effect of TGF- $\beta$ and VEGF on production of basementmembrane-related molecules in peripheral nerve pericytes

\begin{tabular}{lll}
\hline Protein & \multicolumn{2}{l}{ Ratio of target protein production (protein:actin) } \\
\cline { 2 - 3 } & TGF- $\beta$ & VEGF \\
\hline Collagen type IV & $4.49 \pm 0.15^{* *}$ & $1.94 \pm 0.20^{* *}$ \\
Fibronectin & $3.60 \pm 0.18^{* *}$ & $1.92 \pm 0.15^{* *}$ \\
TIMP-1 & $1.23 \pm 0.14$ & $1.10 \pm 0.14$ \\
Pro-MMP-9 & $1.84 \pm 0.13^{* *}$ & $3.22 \pm 0.23^{* *}$ \\
Act-MMP-9 & $1.28 \pm 0.14$ & $1.38 \pm 0.17$ \\
Pro-MMP-2 & $0.95 \pm 0.14$ & $1.34 \pm 0.25$ \\
Act-MMP-2 & $1.43 \pm 0.32$ & $1.32 \pm 0.14$ \\
\hline
\end{tabular}

Data shown are means \pm SEM $(n=5)$

Each value reflects the combined densitometry data from five independent experiments and is shown as a fold increase above control

$* * p<0.01$ compared with control 

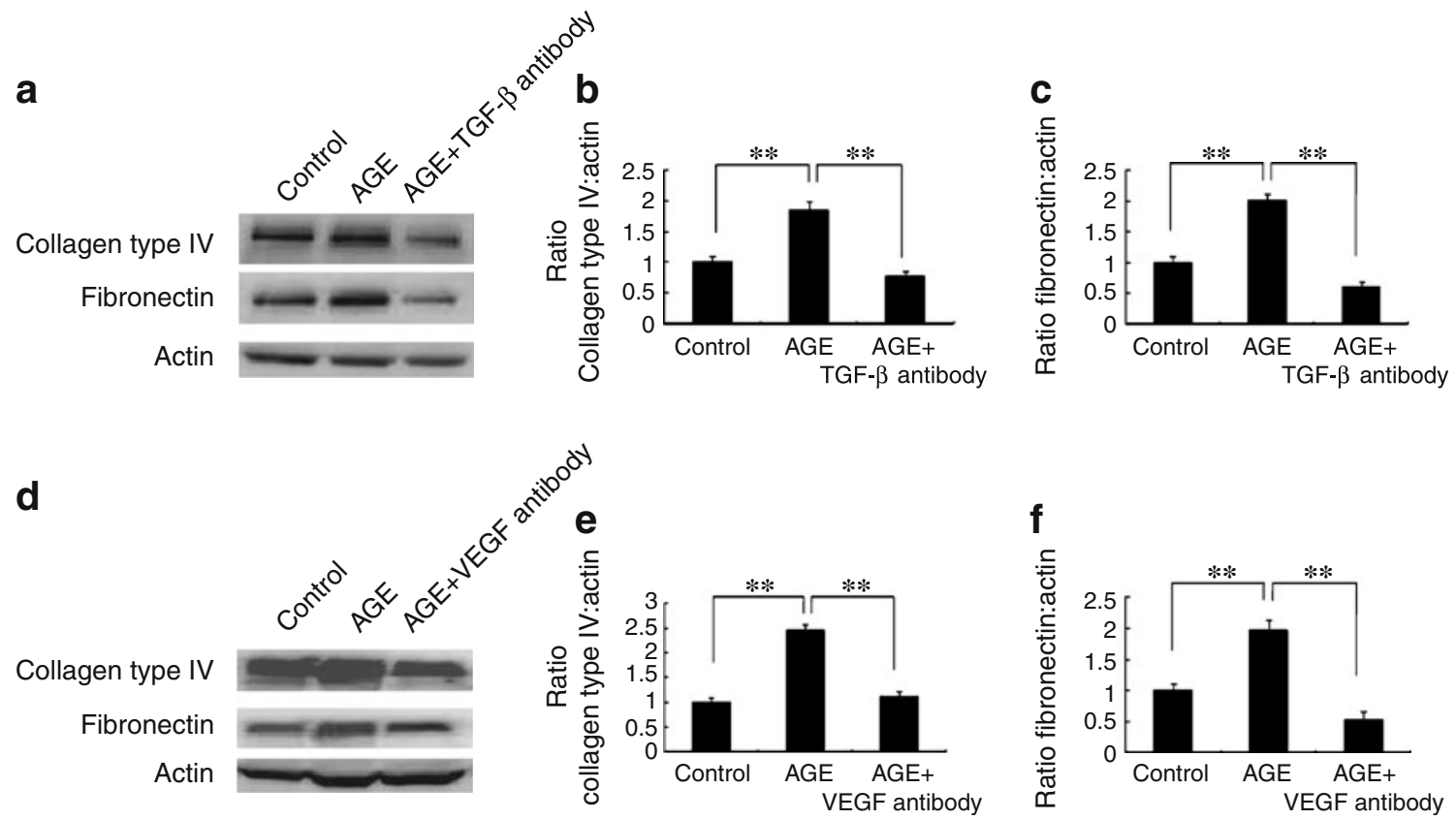

Fig. 4 The effects of anti-TGF- $\beta$ and anti-VEGF neutralising antibodies on the collagen type IV and fibronectin induction by treatment with AGEs. The peripheral nerve pericytes were cultured with AGEs or AGEs pre-treated with anti-TGF- $\beta$ or anti-VEGF antibody for $24 \mathrm{~h}$. a The change in the production of collagen type IV and fibronectin protein in PnMECs after treatment with anti-TGF- $\beta$ neutralising antibody using a western blot analysis. b Collagen type IV and (c) fibronectin proteins were inhibited after pretreatment with anti-TGF- $\beta$ neutralising antibody. $\mathbf{d}$ The change in the production of collagen type IV and fibronectin protein in PnMECs after treatment with anti-VEGF neutralising antibody using a western blot analysis. Production of (e) collagen type IV and (f) fibronectin was inhibited after pretreatment with anti-VEGF neutralising antibody. Each bar graph reflects the combined densitometry data from five independent experiments (mean \pm SEM, $n=5$ ). Control, conditioned medium of unmodified BSA; AGEs, conditioned medium of AGEs-BSA; AGE+ VEGF antibody, conditioned medium of AGEs-BSA pre-treated with VEGF neutralising antibody; AGE + TGF- $\beta$ antibody, conditioned medium of AGE-BSA pre-treated with TGF- $\beta$ neutralising antibody; $* * p<0.01$ of the beneficial effects of anti-VEGF agents in the reduction of retinal vascular permeability [23]. We therefore hypothesised that TGF- $\beta$ and VEGF signalling may contribute to the induction of basement membrane hypertrophy and disruption of the BNB. Our study demonstrated that the production of fibronectin and collagen type IV by peripheral nerve pericytes significantly increased after exposure to TGF- $\beta$ or VEGF, and that the amount of proMMP-9 significantly increased. These results indicate that TGF- $\beta$ and VEGF might directly stimulate the production of fibronectin and collagen type IV in peripheral nerve pericytes, and that the induction of pro-MMP-9 might then occur secondary to these signals, thus leading to degradation of the increased fibronectin or collagen type IV in order to maintain ECM turnover.

AGEs are the late products of non-enzymatic glycation, and their accumulation on proteins in the microvasculature appears to be a key factor in the development of neuropathy [24]. AGEs accumulate in the vessel walls and have been implicated in both the macrovascular and microvascular complications of diabetes [12]. The accumulation of AGEs in both the peripheral nerves of human diabetic patients and in the experimental diabetic animals was observed in vascular endothelial cells, pericytes, and the basement membrane, as well as in axons and Schwann cells, thus resulting in an impaired nerve function and characteristic pathological alterations [24, 25]. Several lines of evidences suggest that AGEs induce basement-membrane thickening in diabetic nephropathy and retinopathy $[13,26]$. However, the molecular mechanism underlying this process is unclear in diabetic neuropathy. Our study demonstrates that the production of collagen type IV and fibronectin in peripheral nerve pericytes was significantly increased after exposure to AGEs. These results thus indicate that AGEs directly induce basement membrane hypertrophy at the BNB.

Several other studies suggest that an imbalance between MMPs and TIMPs may cause basement membrane hypertrophy through inhibition of ECM degradation $[9,13]$. Our results demonstrated that induction of TIMP-1 and reduction of MMP-9 in peripheral nerve pericytes was observed after application of AGEs, thus suggesting that AGEs inhibit ECM degradation at the BNB. Furthermore, our study also demonstrated that the production of VEGF and TGF- $\beta$ secreted from peripheral nerve pericytes was 


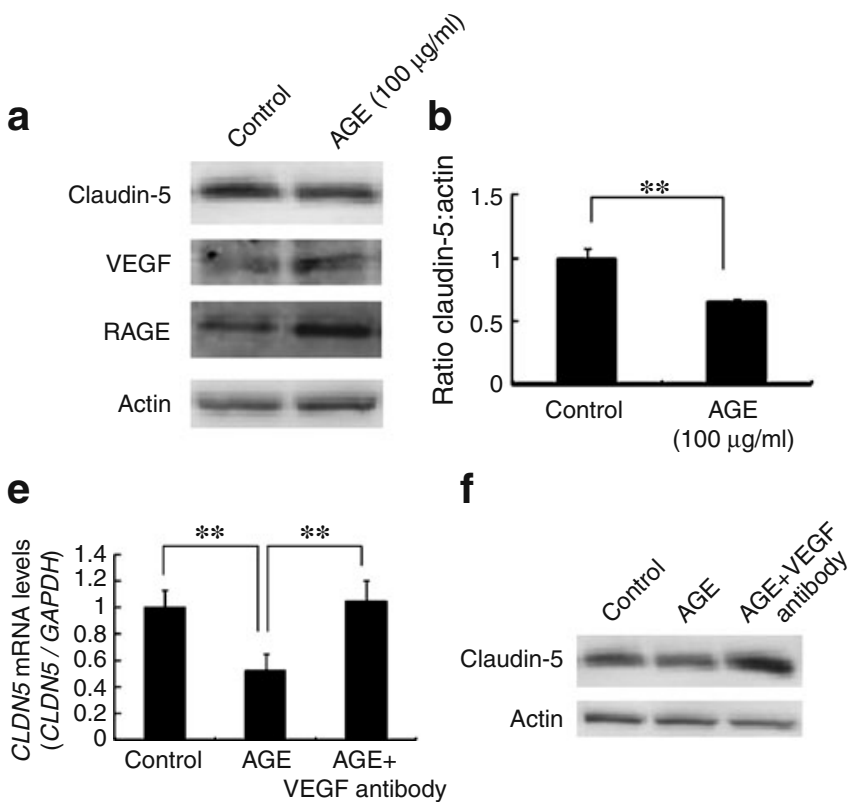

Fig. 5 a The changes in claudin-5, VEGF and RAGE production in peripheral nerve pericytes after treatment with AGEs $(100 \mu \mathrm{g} / \mathrm{ml})$ using western blot analysis. b Claudin-5 in PnMECs was significantly decreased after treatment with AGE. VEGF (c) and (d) RAGE production was significantly increased after incubation with AGE. e The effect of anti-VEGF neutralising antibody on the CLDN5 induction by AGE treatment. The PnMECs were cultured with AGEs or AGEs pre-treated with anti-VEGF antibody for $24 \mathrm{~h}$. The level of CLDN5 mRNA in PnMECs was assayed by real-time RT-PCR and expressed as the ratio of the target gene/GAPDH. The CLDN5 mRNA was upregulated by $48 \%$ following exposure to AGEs pretreated with anti-VEGF antibody as compared with AGEs only. $\mathbf{f}$ The change in

increased after incubation with AGEs. The experimental inhibition studies using anti-VEGF or TGF- $\beta$ neutralising antibodies in peripheral nerve pericytes also revealed that VEGF and TGF- $\beta$ were primarily responsible for the upregulation of fibronectin and collagen type IV. These results indicate that AGEs induce basement membrane hypertrophy and disrupt the BNB by increasing the production of secreted VEGF and TGF- $\beta$ by pericytes under diabetic conditions.

The breakdown of the BRB occurs in patients with diabetic retinopathy [27]. Several studies have suggested that AGEs induce vasopermeability of the retina by increasing VEGF production [14, 23, 28-31] and disrupt the BRB by decreasing tight junction proteins, such as occludin and ZO-1 [32]. However, the role of AGEs in diabetic neuropathy remains unclear. In this study, we examined whether AGEs could alter the integrity of the tight junction in endothelial cells during diabetic neuropathy. Claudin-5 is known to be a major component of tight junctions, and the production of claudin-5 is important for tight junction maintenance in the mature BBB [33]. Our results demonstrate that claudin-5 in PnMECs was
C

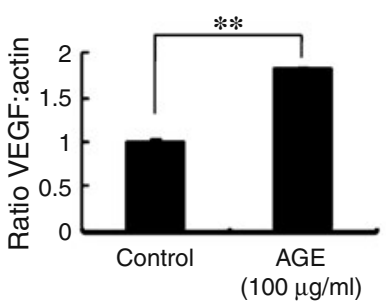

g

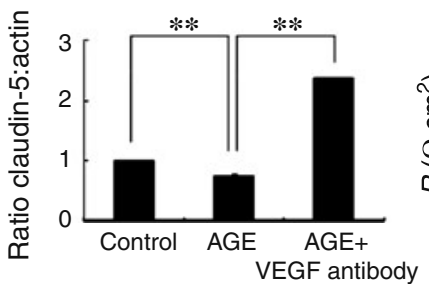

d

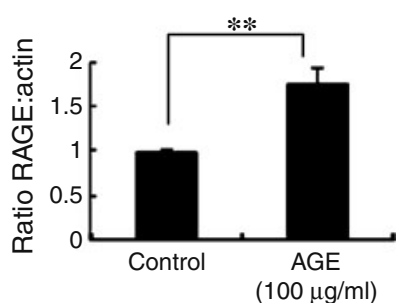

h

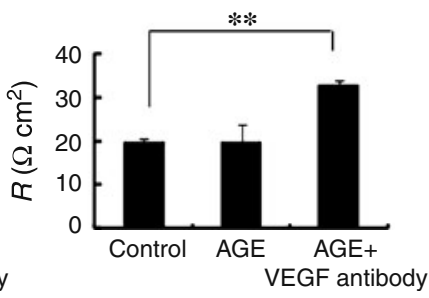

the production of claudin-5 protein in PnMECs after treatment with anti-VEGF neutralising antibody using western blot analysis. g Claudin-5 protein was increased after pretreatment with anti-VEGF neutralising antibody. $\mathbf{h}$ The TEER value of PnMECs was also significantly increased after treatment with AGEs pretreated with antiVEGF neutralising antibody. Each bar graph reflects the combined densitometry data from five independent experiments (mean $\pm \mathrm{SEM}$, $n=5$ ). Control, conditioned medium of unmodified BSA; AGEs, conditioned medium of AGEs-BSA; AGE+VEGF antibody, a conditioned medium of AGE-BSA pre-treated with VEGF neutralising antibody. $* * p<0.01$

decreased after exposure to AGEs. In contrast, the production of VEGF in PnMECs was increased after incubation with AGEs, and our inhibition studies using an anti-VEGF neutralising antibody also revealed that VEGF was primarily responsible for the downregulation of claudin-5. These results indicate that AGEs reduce the production of claudin-5 in PnMECs by increasing the secretion of VEGF secreted by the PnMECs themselves.

Our findings show that the production of RAGE was significantly increased in peripheral nerve pericytes and PnMECs after exposure to AGEs. RAGE is a $35 \mathrm{kDa}$ polypeptide of the Ig superfamily that plays a critical role in the development of diabetic vascular complications [34, 35]. RAGE is present in minimal quantities in the normal endothelial cells and pericytes of the BNB, but is upregulated in a positive-feedback manner when AGE ligands accumulate [24, 36]. These activities may induce inflammatory responses, leading to aggravation of diabetic vascular complications [36]. RAGE has also been reported to activate transcription factor nuclear factor $\mathrm{KB}$ cells (NF$\mathrm{KB}$ ), thus leading to increase production of the cytokines including TNF- $\alpha[34,35]$. Several studies have demon- 
strated that NF- $\mathrm{kB}$ activation may induce VEGF and TGF- $\beta$ production $[37,38]$. The activation of RAGE in PnMECs and peripheral nerve pericytes after exposure to AGEs may thus induce the production of VEGF and TGF- $\beta$ through NF- $\mathrm{KB}$ signalling. The prevention or decrease of glycation and glycation-induced tissue damage could be possible therapeutic strategies for the treatment of diabetic neuropathy [39, 40]. Recent clinical studies have shown that AGE breakers, including ALT-711 and alagebrium, may be able to decrease the adverse vascular effects of glycation with few side effects [41-43], although no benefits have been demonstrated in the area of neuropathy. The inhibition of pathological responses mediated by AGEs may have therapeutic potential for diabetic neuropathy, although the RAGE-mediated molecular mechanisms need to be explored in further studies.

In conclusion, we have herein demonstrated that pericytes are the main regulators involved in the maintenance of the basement membrane at the BNB, and that AGEs induce basement membrane hypertrophy and disrupt the BNB by increasing the secretion of VEGF and TGF- $\beta$ released by pericytes and the secretion of VEGF secreted by PnMECs under diabetic conditions. Further research is necessary because obtaining a better understanding of the molecular mechanisms by which AGEs induce BNB breakdown is expected to provide new targets for the treatment and prevention of diabetic neuropathy.

Acknowledgements This work was supported by research grants (numbers 22790821 and 21390268) from the Japan Society for the Promotion of Science, Tokyo, Japan and also by research grant (K2002528) from Health and Labor Sciences Research Grants for research on intractable diseases (Neuroimmunological Disease Research Committee) from the Ministry of Health, Labor and Welfare of Japan.

Duality of interest The authors declare that there is no duality of interest associated with this manuscript.

\section{References}

1. Abbott NJ, Rönnbäck L, Hansson E (2006) Astrocyte-endothelial interactions at the blood-brain barrier. Nat Rev Neurosci 7:41-53

2. Poduslo JF, Curran GL, Berg CT (1994) Macromolecular permeability across the blood-nerve and blood-brain barriers. Proc Natl Acad Sci USA 91:5705-5709

3. Sano Y, Shimizu F, Nakayama H, Abe M, Maeda T, Ohtsuki S, Terasaki T, Obinata M, Ueda M, Takahashi R, Kanda T (2007) Endothelial cells constituting blood-nerve barrier have highly specialized characteristics as barrier-forming cells. Cell Struct Funct 32:139-147

4. Vinik AI, Mehrabyan A (2004) Diabetic neuropathies. Med Clin North Am 88:947-999

5. Cameron NE, Eaton SE, Cotter MA, Tesfaye S (2001) Vascular factors and metabolic interactions in the pathogenesis of diabetic neuropathy. Diabetologia 44:1973-1988

6. Giannini C, Dyck PJ (1995) Basement membrane reduplication and pericyte degeneration precede development of diabetic polyneuropathy and are associated with its severity. Ann Neurol $37: 498-504$

7. Grant DS, Kleinman HK (1997) Regulation of capillary formation by laminin and other components of the extracellular matrix. EXS 79:317-333

8. Tilling T, Engelbertz C, Decker S, Korte D, Hüwel S, Galla HJ (2002) Expression and adhesive properties of basement membrane proteins in cerebral capillary endothelial cell cultures. Cell Tissue Res 310:19-29

9. Davis GE, Senger DR (2005) Endothelial extracellular matrix: biosynthesis, remodeling, and functions during vascular morphogenesis and neovessel stabilization. Circ Res 97:1093-1107

10. Jiang B, Liou GI, Behzadian MA, Caldwell RB (1994) Astrocytes modulate retinal vasculogenesis: effects on fibronectin expression. J Cell Sci 107:2499-2508

11. Kose N, Asashima T, Muta M, Iizasa H, Sai Y, Terasaki T, Nakashima E (2007) Altered expression of basement membranerelated molecules in rat brain pericyte, endothelial, and astrocyte cell lines after transforming growth factor-beta1 treatment. Drug Metab Pharmacokinet 22:255-266

12. Goldin A, Beckman JA, Schmidt AM, Creager MA (2006) Advanced glycation end products: sparking the development of diabetic vascular injury. Circulation 114:597-605

13. Ban CR, Twigg SM (2008) Fibrosis in diabetes complications: pathogenic mechanisms and circulating and urinary markers. Vasc Health Risk Manag 4:575-596

14. Canning P, Glenn JV, Hsu DK, Liu FT, Gardiner TA, Stitt AW (2007) Inhibition of advanced glycation and absence of galectin-3 prevent blood-retinal barrier dysfunction during short-term diabetes. Exp Diabetes Res: 51837

15. Shimizu F, Sano Y, Abe MA, Maeda T, Ohtsuki S, Terasaki T, Obinata M, Kanda T (2011) Peripheral nerve pericytes modify the blood-nerve barrier function and tight junctional molecules through the secretion of various soluble factors. J Cell Physiol 226:255-266

16. Shimizu F, Sano Y, Maeda T, Abe MA, Nakayama H, Takahashi R, Ueda M, Ohtsuki S, Terasaki T, Obinata M, Kanda T (2008) Peripheral nerve pericytes originating from the blood-nerve barrier expresses tight junctional molecules and transporters as barrier-forming cells. J Cell Physiol 217:388-399

17. Muir EM, Adcock KH, Morgenstern DA, Clayton R, von Stillfried N, Rhodes K, Ellis C, Fawcett JW, Rogers JH (2002) Matrix metalloproteases and their inhibitors are produced by overlapping populations of activated astrocytes. Brain Res Mol Brain Res 100:103-117

18. Ziyadeh FN, Han DC, Cohen JA, Guo J, Cohen MP (1998) Glycated albumin stimulates fibronectin gene expression in glomerular mesangial cells: involvement of the transforming growth factor-beta system. Kidney Int 53:631-638

19. Sharma K, Ziyadeh FN (1995) Hyperglycemia and diabetic kidney disease. The case for transforming growth factor-beta as a key mediator. Diabetes 44:1139-1146

20. Sharma K, Jin Y, Guo J, Ziyadeh FN (1996) Neutralization of TGF-beta by anti-TGF-beta antibody attenuates kidney hypertrophy and the enhanced extracellular matrix gene expression in STZinduced diabetic mice. Diabetes 45:522-530

21. Ziyadeh FN, Hoffman BB, Han DC, Iglesias-De La Cruz MC, Hong SW, Isono M, Chen S, McGowan TA, Sharma K (2000) Long-term prevention of renal insufficiency, excess matrix gene expression, and glomerular mesangial matrix expansion by treatment with monoclonal antitransforming growth factor-beta antibody in $\mathrm{db} / \mathrm{db}$ diabetic mice. Proc Natl Acad Sci USA 97:8015-8020

22. Sung SH, Ziyadeh FN, Wang A, Pyagay PE, Kanwar YS, Chen S (2006) Blockade of vascular endothelial growth factor signaling ameliorates diabetic albuminuria in mice. J Am Soc Nephrol 17:3093-3104 
23. Penn JS, Madan A, Caldwell RB, Bartoli M, Caldwell RW, Hartnett ME (2008) Vascular endothelial growth factor in eye disease. Prog Retin Eye Res 27:331-371

24. Wada R, Yagihashi S (2005) Role of advanced glycation end products and their receptors in development of diabetic neuropathy. Ann NY Acad Sci 1043:598-604

25. Sugimoto K, Nishizawa Y, Horiuchi S, Yagihashi S (1997) Localization in human diabetic peripheral nerve of $\mathrm{N}$ (epsilon)carboxymethyllysine-protein adducts, an advanced glycation endproduct. Diabetologia 40:1380-1387

26. Tsilibary EC (2003) Microvascular basement membranes in diabetes mellitus. J Pathol 200:537-546

27. Khan ZA, Chakrabarti S (2007) Cellular signaling and potential new treatment targets in diabetic retinopathy. Exp Diabetes Res. 31867

28. Stitt AW, Bhaduri T, McMullen CB, Gardiner TA, Archer DB (2000) Advanced glycation end products induce blood-retinal barrier dysfunction in normoglycemic rats. Mol Cell Biol Res Commun 3:380-388

29. Lu M, Kuroki M, Amano S, Tolentino M, Keough K, Kim I, Bucala R, Adamis AP (1998) Advanced glycation end products increase retinal vascular endothelial growth factor expression. J Clin Invest 101:1219-1224

30. Yamagishi S, Yonekura H, Yamamoto Y, Katsuno K, Sato F, Mita I, Ooka H, Satozawa N, Kawakami T, Nomura M, Yamamoto H (1997) Advanced glycation end products-driven angiogenesis in vitro. Induction of the growth and tube formation of human microvascular endothelial cells through autocrine vascular endothelial growth factor. J Biol Chem 272:8723-8730

31. McFarlane S, Glenn JV, Lichanska AM, Simpson DA, Stitt AW (2005) Characterisation of the advanced glycation endproduct receptor complex in the retinal pigment epithelium. $\mathrm{Br} \mathrm{J}$ Ophthalmol 89:107-112

32. Sheikpranbabu S, Kalishwaralal K, Lee KJ, Vaidyanathan R, Eom SH, Gurunathan S (2010) The inhibition of advanced glycation end-products-induced retinal vascular permeability by silver nanoparticles. Biomaterials 31:2260-2271
33. Nitta T, Hata M, Gotoh S, Seo Y, Sasaki H, Hashimoto N, Furuse M, Tsukita S (2003) Size-selective loosening of the blood-brain barrier in claudin-5-deficient mice. J Cell Biol 161:653-660

34. Farmer DG, Kennedy S (2009) RAGE, vascular tone and vascular disease. Pharmacol Ther 124:185-194

35. Yonekura H, Yamamoto Y, Sakurai S, Watanabe T, Yamamoto $H$ (2005) Roles of the receptor for advanced glycation endproducts in diabetes-induced vascular injury. J Pharmacol Sci 97:305-311

36. Lukic IK, Humpert PM, Nawroth PP, Bierhaus A (2008) The RAGE pathway: activation and perpetuation in the pathogenesis of diabetic neuropathy. Ann NY Acad Sci 1126:76-80

37. Djordjević G, Matusan-Ilijas K, Sinozić E, Damante G, Fabbro D, Grahovac B, Lucin K, Jonjić N (2008) Relationship between vascular endothelial growth factor and nuclear factor-kappaB in renal cell tumors. Croat Med J 49:608-617

38. Saile B, Matthes N, El Armouche H, Neubauer K, Ramadori G (2001) The bcl, NFkappaB and p53/p21WAF1 systems are involved in spontaneous apoptosis and in the anti-apoptotic effect of TGF-beta or TNF-alpha on activated hepatic stellate cells. Eur J Cell Biol 80:554-561

39. Huijberts MS, Schaper NC, Schalkwijk CG (2008) Advanced glycation end products and diabetic foot disease. Diabetes Metab Res Rev 24:S19-S24

40. Monnier VM (2003) Intervention against the Maillard reaction in vivo. Arch Biochem Biophys 419:1-15

41. Kass DA, Shapiro EP, Kawaguchi M, Capriotti AR, Scuteri A, deGroof RC, Lakatta EG (2001) Improved arterial compliance by a novel advanced glycation end-product crosslink breaker. Circulation 104:1464-1470

42. Little WC, Zile MR, Kitzman DW, Hundley WG, O’Brien TX, Degroof RC (2005) The effect of alagebrium chloride (ALT-711), a novel glucose cross-link breaker, in the treatment of elderly patients with diastolic heart failure. J Card Fail 11:191-195

43. Zieman SJ, Melenovsky V, Clattenburg L, Corretti MC, Capriotti A, Gerstenblith G, Kass DA (2007) Advanced glycation endproduct crosslink breaker (alagebrium) improves endothelial function in patients with isolated systolic hypertension. J Hypertens 25:577-583 